\title{
Cooperative functioning of unconscious and consciousness from theory of open quantum systems
}

\author{
Andrei Khrennikov \\ Linnaeus University, International Center for Mathematical Modeling \\ in Physics and Cognitive Sciences Växjö, SE-351 95, Sweden
}

January 20, 2021

\begin{abstract}
We present the mathematical model of cooperative functioning of unconscious and consciousness. The model is based on the theory of open quantum systems. Unconscious and consciousness are treated as bio-information systems. The latter plays the role a measurement apparatus for the former. States of both systems are represented in Hilbert spaces. Consciousness performs measurements on the states which are generated in unconscious. This process of unconscious-conscious interaction is described by the scheme of indirect measurements. This scheme is widely used in quantum information theory and it leads to the theory of quantum instruments (Davis-Lewis-Ozawa). Our approach is known as quantum-like modeling. It should be sharply distinguished from modeling of genuine quantum physical processes in biosystems, in particular, in the brain. In the quantum-like framework, the brain is a black box processing information in the accordance with the laws of quantum theory. During the last 10-15 years this framework has been actively used in cognition, psychology, decision making, social and political sciences. The quantum-like scheme of unconscious-consciousness functioning has already been explored for sensation-perception modeling.
\end{abstract}

keywords: unconscious, consciousness, quantum-like models, decision making, indirect measurement scheme, open quantum systems, sensation, perception.

\section{Introduction}

The quantum information revolution (also known as the second quantum revolution) has big impact not only to technology, but also to quantum foundations. This revolution led 
to elaboration of few information interpretations of quantum mechanics (see, e.g., [1][11] and some papers in proceedings' volumes [12]- [16]). By these interpretations, the physical properties of the carriers and processors of quantum information are not crucial. In particular, the role of the physical space is diminished essentially; the quantum information scenarios is written for the information space. Tremendous development of quantum information theory endowed with experimental and technological applications stimulated its use outside of physics, especially in cognition, psychology, decision making, social and political science, economics and finance see pioneer works [17][19], monographs [20]- [25] and some papers [26]- [64]. Such modeling is known as quantum-like.

Quantum-like systems (proteins, cells, body's organs, including the brain, human beings, social, political, economic, and financial systems) represent and process information by operating with quantum information states. Such quantum-like, i.e., respecting the quantum laws, information processing has no direct connection with the genuine quantum physical processes in a biological organism. A biosystem is a black box performing quantum information processing. The ability to represent information in the quantum-like way was developed in the process of evolution of biosystems. It has no direct relation with system's spatial and temporal scales, temperature and other parameters of this sort (cf. [65]- [69]). In article [50], it was pointed out that the quantum representation of information is the distinguishing feature of all biosystems, from proteins, genomes, and cells to brains and ecological systems. One can speak about quantum information biology.

What is the main feature of the quantum-like representation and processing?

It is the possibility of processing unresolved uncertainties. Mathematically, they are encoded in states' superpositions. What is the main advantage of such information processing (operating with superpositions)? It saves computational resources: biosystems working in the quantum-like regime need not resolve all uncertainties and determine outcomes of variables and their probability distributions at each step of state's processing. The outcomes are determined in the process of measurement. Measurements are performed by measurement apparatuses. The quantum formalism is general calculus describing processing of superpositions and extraction of the variables' values - measurements. The most powerful quantum measurement formalism is based on the theory of open quantum systems [70,71]: a system $S$ interacting with the surrounding environment $E$. In the particular application of this theory to the description of measurements, the role of environment is played by a measurement apparatus $M$ used to measure some observable $A$ on $S$. One of the basic mathematical frameworks for modeling of this situation is the scheme of indirect measurements [72]. The outcomes of $A$ are represented as outcomes of apparatus' pointer $M_{A}$.

The process of measurement is described as interaction between the system $S$ and the apparatus $M$. This interaction generates the dynamics of the state of the compound 
system $S+M$. The state's evolution is unitary; for pure states, it is described by the Schrödinger equation (for mixed states given by density operators, by the von Neumann equation). Finally, the probability distribution for pointer's outcomes is extracted from the compound state with the trace-operation.

For our model, it is important that in this measurement scheme the features of the system $S$ are not approachable directly, but only through apparatus' pointer. We shall explore this in modeling of brain's functioning.

In this paper we consider the quantum-like model in that unconscious $\mathcal{U C}$ and consciousness $\mathcal{C}$ are represented as the system $S$ and the apparatus $M$ in the above indirect measurement scheme. Features of unconscious $\mathcal{U C}$ are inapproachable directly. Consciousness $\mathcal{C}$ performs measurements on the bio-information system $\mathcal{U C}$. The main difference from physics is that to make a decision $\mathcal{C}$ should "read its pointer" by itself. So, $\mathcal{U C}$ performs self-measurements. However, the latter does not change the formal mathematical scheme.

This is the good place to remark that even in quantum physics the pointer-reading is the nontrivial step of the measurement process. However, typically it is ignored, even in the foundational discussions. We just remark that Wigner claimed that consciousness is really involved in quantum measurement's finalization. (Wigner's viewpoint was not accepted by the majority of physicists.)

This paper is a concept paper. Our aim is to describe the very general scheme of cooperative functioning of unconscious $\mathcal{U C}$ and consciousness $\mathcal{C}$. The scheme is simple and easily understandable. Its concrete application to quantum-like modeling of the sensation-perception process [73] was presented in [52]. However, paper [52] is mathematically complicated, it is based on the theory of quantum instruments $[72,74,75]$. The indirect measurements and quantum instruments are closely related, but in this paper we shall not discuss this issue (see [76,77] where these quantum tools are jointly presented as simple as possible - to be used by psychologists and biologists).

\section{A few words about quantum formalism}

In quantum theory, it is postulated that every quantum system $S$ corresponds to a complex Hilbert space $\mathcal{H}$; denote the scalar product of two vectors by the symbol $\left\langle\psi_{1} \mid \psi_{2}\right\rangle$. Throughout the present paper, we assume $\mathcal{H}$ is finite dimensional. States of the quantum system $S$ are represented by density operators acting in $\mathcal{H}$ (positive semi-definite operators with unit trace). Denote this state space by the symbol $\mathbf{S}(\mathcal{H})$.

In quantum physics (especially quantum information theory), there are widely used notations invented by Dirac: a vector belonging to $\mathcal{H}$ is symbolically denoted as $|\psi\rangle$; orthogonal projector on this vector is denoted as $|\psi\rangle\langle\psi|$, it acts to the vector $|\xi\rangle$ as $\langle\psi \mid \xi\rangle|\psi\rangle$. 
Any density operator $\rho$ of rank one is of the form $\rho=|\psi\rangle\langle\psi|$ with a unit-norm vector $|\psi\rangle$. In this case, $|\psi\rangle$ is called a pure state, so $|\psi\rangle \in \mathcal{H}, \||\psi\rangle \|=\sqrt{\langle\psi \mid \psi\rangle}=1$.

Observables are represented by Hermitian operators in $\mathcal{H}$. These are just symbolic expressions of phsyical observables, say the position, momentum, or energy. Each Hermitian operator $A$ can be represented as

$$
A=\sum_{x} x E^{A}(x)
$$

where $x$ labels the eigenvalues and $E^{A}(x)$ is the spectral projection of the observable $A$ corresponding to the eigenvalue $x$.

The operator $A$ can be considered as the compact mathematical representation for probabilities of outcomes of the physical observable. These probabilities are given by the Born rule that states if an observable $A$ is measured in a state $\rho$, then the probability distribution $\operatorname{Pr}\{A=x \| \rho\}$ of the outcome of the measurement is given by

$$
\operatorname{Pr}\{A=x \| \rho\}=\operatorname{Tr}\left[E^{A}(x) \rho\right]=\operatorname{Tr}\left[E^{A}(x) \rho E^{A}(x)\right] .
$$

For a pure state $|\psi\rangle$, this leads to the relation

$$
\operatorname{Pr}\{A=x \||\psi\rangle\}=\| E^{A}(x)|\psi\rangle \|^{2},
$$

as $\operatorname{Tr}\left[E^{A}(x) \rho\right]=\| E^{A}(x)|\psi\rangle \|^{2}$.

\section{Indirect measurement scheme: apparatus with meter interacting with a system}

The scheme of indirect measurements represents the framework which was emphasized by Bohr, by him the outcomes of quantum measurements are created in the complex process of the interaction of a system $S$ with a measurement apparatus $M$. The latter is combined of a complex physical device interacting with $S$ and a pointer showing the outcomes of measurements; for example, it can be the "spin up or spin down" arrow. The system $S$ by itself is not approachable by the observer who can see only the pointer of $M$. Then the observer associates pointer's outputs with the values of measured observable $A$ for the system $S$.

Can the outputs of the pointer be associated with the "intrinsic properties" of $S$ or not? This is one of the main questions of disturbing the quantum foundations during the last 100 years.

The indirect measurement scheme can be represented as the block of following interrelated components: 
- the states of the systems $S$ and the apparatus $M$; they are represented in complex Hilbert spaces $\mathcal{H}$ and $\mathcal{K}$, respectively;

- the unitary operator $U$ representing the interaction-dynamics for the compound system $S+M$;

- the meter observable $M_{A}$ giving outputs of the pointer of the apparatus $M$.

In quantum physics, the operator $U$ representing the dynamics of interaction $S$ and $M$ is a linear unitary operator. All operations in quantum theory are linear and dynamics of an isolated system is unitary, i.e., preserving the scalar product. In cognitive applications, we proceed with linear and unitary operators just for simplicity, just to borrow the well-defined formalism and interpretation from physics. In principle, there are no reason to assume neither linearity nor unitarity. We remark that even in physics there were attemps to develop nonlinear quantum theory.

In the indirect measurement scheme, it is assumed that the compound system $S+M$ is isolated. The dynamics of pure states of the compound system is described by the Schrödinger equation:

$$
i \frac{d}{d t}|\Psi\rangle(t)=H|\Psi\rangle(t),|\Psi\rangle(0)=|\Psi\rangle_{0},
$$

where $H$ is it Hamiltonian (generator of evolution) of $S+M$. The state $|\Psi\rangle(t)$ evolves as

$$
|\Psi\rangle(t)=U(t)|\Psi\rangle_{0},
$$

where $U(t)$ is the unitary operator represented as

$$
U(t)=e^{-i t H} .
$$

Hamiltonian (evolution-generator) describing information interactions has the form

$$
H=H_{S} \otimes I+I \otimes H_{M}+H_{S, M},
$$

where $H_{S}: \mathcal{H} \rightarrow \mathcal{H}, H_{M}: \mathcal{K} \rightarrow \mathcal{K}$ are Hamiltonians of $S$ and $M$, respectively, and $H_{S, M} \in \mathcal{H} \otimes \mathcal{K} \rightarrow \mathcal{H} \otimes \mathcal{K}$ is Hamiltonian of interaction between systems $S$ and $M$. The Schrödinger equation implies that evolution of the density operator $R(t)$ of the system $S+M$ is described by the von Neumann equation:

$$
\frac{d R}{d t}(t)=-i[H, R(t)], R(0)=R_{0}
$$

However, the state $R(t)$ is too complex to be handled consistently: the apparatus includes many degrees of freedom. 
Suppose that we want to measure an observable on the system $S$ which is represented by Hemitian operator $A$, acting in system's state space $\mathcal{H}$. The indirect measurement model for measurement of the $A$-observable was introduced by Ozawa in [72] as a "(general) measuring process"; this is a quadruple

$$
\left(\mathcal{K}, \sigma, U, M_{A}\right)
$$

consisting of a Hilbert space $\mathcal{K}$, a density operator $\sigma \in \mathbf{S}(\mathcal{K})$, a unitary operator $U$ on the tensor product of the state spaces of $S$ and $M, U: \mathcal{H} \otimes \mathcal{K} \rightarrow \mathcal{H} \otimes \mathcal{K}$, and a Hermitian operator $M_{A}$ on $\mathcal{K}$.

Here $\mathcal{K}$ represents the states of the apparatus $M, U$ describes the time-evolution of system $S+M, \sigma$ describes the initial state of the apparatus $M$ before the start of measurement, and the Hermitian operator $M_{A}$ is the meter observable of the apparatus $M$ (say the pointer of $M$ ). This operator represents indirectly outcomes of an observable $A$ for the system $S$.

The probability distribution $\operatorname{Pr}\{A=x \| \rho\}$ in the system state $\rho \in \mathbf{S}(\mathcal{H})$ is given by

$$
\operatorname{Pr}\{A=x \| \rho\}=\operatorname{Tr}\left[\left(I \otimes E^{M_{A}}(x)\right) U(\rho \otimes \sigma) U^{\star}\right],
$$

where $E^{M_{A}}(x)$ is the spectral projection of $M_{A}$ for the eigenvalue $x$. We reall that operator $M_{A}$ is Hermitian. In the finite dimensional case, it can be represented in the form:

$$
M_{A}=\sum_{k} x_{k} E^{M_{A}}\left(x_{k}\right)
$$

where $\left(x_{k}\right)$ is the set of its eigenvalues and $E^{M_{A}}\left(x_{k}\right)$ is the projector on the subspace of eigenvectors corresponding to eigenvalue $x_{k}$.

The change of the state $\rho$ of the system $S$ caused by the measurement for the outcome $A=x$ is represented with the aid of the map $\mathcal{I}_{A}(x)$ in the space of density operators defined as

$$
\mathcal{I}_{A}(x) \rho=\operatorname{Tr}_{\mathcal{K}}\left[\left(I \otimes E^{M_{A}}(x)\right) U(\rho \otimes \sigma) U^{\star}\right],
$$

where $\operatorname{Tr}_{\mathcal{K}}$ is the partial trace over $\mathcal{K}$. The map $x \mapsto \mathcal{I}_{A}(x)$ is a quantum instrument. We remark that conversely any quantum instrument can be represented via the indirect measurement model (see Ozawa [72]).

\section{More technical details}

Now, we consider some technicalities. For simplicity, they were not present in the previous section.

The interaction between the system $S$ and the apparatus $M$ starts at time $t_{0}$. The interaction turns off at time $t=t_{0}+\Delta t$. The indirect measurement scheme is based on the following natural assumptions: 
- The system $S$ and the measurement apparatus $M$ do not interact each other before the instant of time $t_{0}$ nor after $t=t_{0}+\Delta t$.

- The compound system $S+M$ is isolated in the time interval $\left(t_{0}, t\right)$.

The latter assumption is too strong. Of course, a macroscopic apparatus $M$ cannot be completely isolated, it interacts with surrounding material bodies and fields. ${ }^{1}$ Therefore by speaking about isolation it is more natural to speak not about the whole apapratus $M$, but just its part interacting with $S$. This situation is formalized as follows.

The probe system $P$ is defined to be the minimal part of apparatus $M$ such that the compound system $S+P$ is isolated in the time interval $\left(t_{0}, t\right)$.

The indirect measurement scheme presented in section 3 is applied to the probe system $P$, instead of the whole apparatus $M$. The rest of the apparatus $M$ performs the pointer measurement on the probe $P$. In particular, the unitary evolution operator $U$ describing the state-evolution of the system $S+P$ has the form $U(t)=e^{-i \Delta t H}$, where $H=H_{S}+H_{P}+H_{S P}$ is Hamiltonian of $S+P$ with the terms $H_{S}$ and $H_{P}$ representing the internal dynamics in the subsystems $S$ and $P$ of the compound system and $H_{S P}$ describing the interaction between the subsystems.

Consideration of probe systems is especially useful in the following situation: the total apparatus $M$ is a macroscopic system that interacts (typically in parallel) with a family of systems $S_{j}, j=1,2, \ldots, m$. Different probes of $M$ interact with the concrete systems, the probe $P_{j}$ with the system $S_{j}$. And the compound system $S_{j}+P_{j}$ can be considered as an isolated system, even from interactions with $S_{i}$ and $P_{i}, i \neq j$.

The indirect measurement scheme is a part of the theory of open quantum systems [70]. Instead of a measurement apparatus $M$, we can consider the surrounding environment $\mathbf{E}$ of the system $S$ (see [25], [34, 36, 37, 50, 55, 57, 60] for applications to psychology).

\section{Indirect measurements of mental observables: Un- conscious as a system and consciousness as a measure- ment apparatus}

The scheme of indirect measurements presented in the previous section was created in quantum physics and applied successfully to a plenty of important problems. Our aim is to adapt it to cognition. The main question is about cognitive analogues of the system $S$ and the measurement apparatus $M$. We suggest to use the framework developed in paper

\footnotetext{
${ }^{1}$ In advanced experimenting in quantum foundations, experimenters put tremendous efforts to isolate their labs. For example, the crucial experiment on Bell's inequa.lity violation (Zeilinger's group [78]) was done in the basement of one of Vienna's castles.
} 
[52] for quantum-like modeling of the Helmholtz sensation-perception theory [73]. This scheme can be extended to a general scheme of unconscious-conscious interaction in the process of decision making

The measured system $S$ is a sensation (or generally any state of the unconscious mind). Consciousness as the measurement apparatus to the unconscious interacts with sensations to make the decisions (to generate outcomes of measurements). Consciousness, of course, is not concerned just with a single probe. It is a large environment with many probes interacting with the unconscious. There should be a rule of transformation from a sensation to a conscious decision. This is unitary transformation and measurement of the meter in the probe. The operational description neglects neurophysiological and electrochemical structures of interaction. The unconscious is a black box that is mathematically described by the state of sensation space (= the unconscious state), so that the unconscious state probabilistically determines the decision (by interaction with consciousness), then the unconscious state is changed according to the previous unconscious state and the decision made. Thus, each probe is described by a quantum instrument. Instruments are probe dependent.

For the question-measurements, the question $A$ is transferred into the unconscious, where it plays the role of a sensation (cf. [73]), so to say a high mental level sensation. Then, interaction described by the unitary operator $U$ generates a new state of the compound system - the unconscious-conscious. And consciousness performs the final "pointer reading", the measurement of the meter observable. Pointer reading can be treated as generation of a perception, a high mental level perception.

\section{Contextuality}

The scheme of indirect measurements formalizes contextuality of conscious-observations. For the fixed state $\rho$, obseravtion's context is determined by triple $C_{A}=\left(\sigma, U, M_{A}\right)$, where, as in the above consideration, $\sigma \in \mathbf{S}(\mathcal{K})$ is the state of $\mathcal{C}$ and $U$ is the unitary operator representing $\rho-\sigma$ interaction and $M_{A}$ represents apparatus' pointer. The same observable $A$ (say question or task) can be realized in various contexts corresponding to variety of states of $\mathcal{C}$ and $\mathcal{U C}-\mathcal{C}$ interactions. The only constraint determining the class of contexts generated by $\mathcal{C}$ is the probability distribution of $A$, given by equality (6), i.e., two contexts for $A$-observable, $C_{A}$ and $C_{A}^{\prime}$ are coupled by the equality:

$$
\operatorname{Pr}\{A=x \| \rho\}=\operatorname{Tr}\left[\left(I \otimes E^{M_{A}}(x)\right) U(\rho \otimes \sigma) U^{\star}\right]=\operatorname{Tr}\left[\left(I \otimes E^{M_{A}^{\prime}}(x)\right) U^{\prime}(\rho \otimes \sigma) U^{\prime \star}\right]
$$

We remark that the state space $\mathcal{K}$ can also vary and it can be considered as a variable determining context. However, we shall not separately emphasize the role of variability of state space $\mathcal{K}$ and assume that this variable is associated with the interaction-operator $U$ and state $\sigma$. 
Context determines the state transformation (8) that has to be denoted not simply as $\mathcal{I}_{A}(x)$, but as $\mathcal{I}_{C_{A}}(x)$. Depending on context $C_{A}, \mathcal{C}$ can generate different state transformations in $\mathcal{U C}$ caused by the measurement. State transformation can be detected with successive measurement of another observable $B$.

As was shown in $[63,76]$, some psychological effects can provide at least partial information on the form of the quantum instrument $\mathcal{I}_{C_{A}}(x)$, in particular, to exclude von Neumann-Lüders instruments given by state trnasformations of the projection form.

\section{Concluding remarks}

We understand that appealing to the unconscious-conscious description of cognitive proceses is not so common in the modern psychology. However, this description well matches the indirect measurement scheme. We can appeal to the authority of James [79] (appealing to Freud [81] might generate a negative reaction), see also Jung [80]. We also can mention the series of works on the use of the unconscious-conscious scheme in quantum-like modeling of cognition $[17,82,83] .{ }^{2}$ In any event, the scheme of indirect measurements in quantum theory matches well with unconscious-conscious structuring of human mind and decision making as indirect observations on inapproachable bioinformation system, unconscious.

\section{Acknowledgments}

The author would like to thank Irina Basieva and Masanao Ozawa for discussions.

\section{References}

[1] Fuchs, C. A. Quantum mechanics as quantum information (and only a little more), in A. Khrennikov (ed.), Quantum theory: Reconsideration of foundations (Växjö University Press, Växjö), 2002, pp. 463-543.

[2] Khrennikov, A. (2002). Växjö interpretation of quantum mechanics, in Quantum Theory: Reconsideration of Foundations, Ser. Math. Modelling (Växjö Univ. Press), Vol. 2, pp. 163-170; Preprint: arXiv:quant-ph/0202107.

\footnotetext{
${ }^{2}$ See also the series of papers [84]- [87] on modeling of cooperative unconscious-conscious functioning by using the threelike geometry of $p$-adic numbers. Although this model is not quantum, the use of $p$-adic numbers gives the possibility to model discreteness.
} 
[3] Khrennikov, A. (2005). Reconstruction of quantum theory on the basis of the formula of total probability, In: Foundations of probability and physics-3, AIP Conf. Proc., 750 (Amer. Inst. Phys., Melville, NY), pp. 187-218.

[4] Jaeger, G. Quantum information. An overview (Heidelberg-Berlin-New York, Springer, 2007).

[5] Plotnitsky, A. Epistemology and probability: Bohr, Heisenberg, Schrödinger and the nature of quantum-theoretical thinking (Springer, Berlin-New York, 2009).

[6] Khrennikov, A. (2009). Contextual Approach to Quantum Formalism, (Springer, Berlin-Heidelberg-New York).

[7] Fuchs, C. A. and Schack, R. (2011). A Quantum-Bayesian Route to QuantumState Space, Found. Phys. 41, p. 345.

[8] Fuchs, C. A. (2012). QBism, the perimeter of Quantum Bayesianism, arXiv:1003.5209 [quant-ph].

[9] Chiribella, G., D’Ariano, G. M. \& Perinotti, P. Probabilistic theories with purification. Phys. Rev. A 81, 062348 (2012).

[10] Chiribella, G., D’Ariano, G. M. \& Perinotti, P. Informational axioms for quantum theory. In: Foundations of probability and physics - 6 1424, pp. 270-279 (AIP, Melville, NY, 2012).

[11] D'Ariano, G. M. Quantum theory from first principles (An informational approach) (Cambridge Univ. Press, Cambridge, 2019).

[12] Khrennikov, A. (ed.) Quantum theory: Reconsideration of foundations (Växjö Univ. Press, Växjö, 2002).

[13] Adenier, G., Khrennikov, A. and Nieuwenhuizen, Th. M. (eds.) Quantum theory: Reconsideration of foundations-3 810 (AIP, Melville, NY, 2006).

[14] Khrennikov, A. Yu. (ed.) Quantum theory: reconsideration of foundations-2 (Växjö Univ. Press, Växjö, 2004).

[15] Khrennikov, A. (ed.) (2005). Foundations of Probability and Physics-3, American Institute of Physics, Ser. Conference Proceedings 750, Melville, NY.

[16] M. D'Ariano, F. Shao-Ming, E. Haven, B. C. Hiesmayr, A. Yu. Khrennikov, J.A. Larsson, Foundations of probability and physics - 61424 (AIP, Melville, NY, 2012). 
[17] Khrennikov, A. Classical and quantum mechanics on information spaces with applications to cognitive, psychological, social and anomalous phenomena, Found. Physics 29, 1065-1098 (1999).

[18] Khrennikov, A. Quantum-like formalism for cognitive measurements. Biosystems 70, 211-233 (2003).

[19] Khrennikov, A. On quantum-like probabilistic structure of mental information, Open Systems and Information Dynamics 11 (3), 267-275 (2004).

[20] Khrennikov, A. Information dynamics in cognitive, psychological, social, and anomalous phenomena, Ser.: Fundamental Theories of Physics (Kluwer, Dordreht, 2004).

[21] Khrennikov, A. Ubiquitous quantum structure: from psychology to finances (Springer, Berlin-Heidelberg-New York, 2010).

[22] Busemeyer, J. \& Bruza, P. Quantum models of cognition and decision (Cambridge Univ. Press, Cambridge, 2012).

[23] Haven, E. \& Khrennikov, A. Quantum social science (Cambridge Univ. Press, Cambridge, 2013).

[24] Haven, E., Khrennikov, A. and Robinson, T. R. Quantum methods in social science: A first course (WSP, Singapore, 2017).

[25] Asano, M., Khrennikov, A., Ohya, M., Tanaka, Y. \& Yamato, I. Quantum Adaptivity in biology: from genetics to cognition (Springer, Heidelberg-Berlin-New York, 2015).

[26] Haven, E. Pilot-wave theory and financial option pricing. Int. J. Theor. Phys. 44 (11), 1957-1962 (2005).

[27] Khrennikov, A. Quantum-like brain: Interference of minds BioSystems 84, 225241 (2006).

[28] Busemeyer, J. R., Wang, Z. \& Townsend, J. T. Quantum dynamics of human decision making. J. Math. Psych. 50, 220-241 (2006).

[29] Choustova O. (2007). Quantum Bohmian model for financial market, Physica A: Statistical Mechanics and its Applications, 374(1), 304-314.

[30] Choustova, O. (2008). Application of Bohmian mechanics to dynamics of prices of shares: Stochastic model of Bohm-Vigier from properties of price trajectories, Int. J. Theor. Phys. 47(1), 252-260. 
[31] Choustova, O. (2009). Quantum probability and financial market, Information Sciences, 179(5), 478-484.

[32] Pothos, E. \& Busemeyer J. R. A quantum probability explanation for violations of 'rational' decision theory. Proceedings of Royal Society B 276, 2171-2178 (2009).

[33] Yukalov, V.I. \& Sornette, D. Physics of risk and uncertainty in quantum decision making. Eur. Phys. J. B 71, 533-548 (2009).

[34] Asano, M. Ohya, M., Tanaka, Y., Basieva, I. \& Khrennikov, A. Quantum-like model of brain's functioning: decision making from decoherence. J. Theor. Biology 281(1), 56-64 (2011).

[35] Dzhafarov, E. N. \& Kujala, J. V. Selectivity in probabilistic causality: Where psychology runs into quantum physics. J. Math. Psych. 56, 54-63 (2012).

[36] Asano, M., Basieva, I., Khrennikov, A,, Ohya, M., Tanaka, Y, \& Yamato, I. Quantum-like model for the adaptive dynamics of the genetic regulation of $\mathrm{E}$. coli's metabolism of glucose-lactose. Syst. Synth. Biol 6, 1-7 (2012).

[37] Asano, M., Basieva, I., Khrennikov, A,, Ohya, M., Tanaka, Y, \& Yamato, I. Towards modeling of epigenetic evolution with the aid of theory of open quantum Systems. 1508, pp. 75-85 (AIP, Melville, NY, 2012); doi.org/10.1063/1.4773118

[38] Bagarello, F. \& Oliveri, F. A phenomenological operator description of interactions between populations with applications to migration. Mathematical Models Meth. Appl. Sc. 23 (03), 471-492 (2013).

[39] Wang, Z. \& Busemeyer, J. R. A quantum question order model supported by empirical tests of an a priori and precise prediction. Top. Cogn. Sc. 5, 689-710 (2013).

[40] Wang, Z., Solloway, T., Shiffrin, R. M. \& Busemeyer, J. R. Context effects produced by question orders reveal quantum nature of human judgments. PNAS 111, 9431-9436 (2014).

[41] Khrennikov, A. \& Basieva, I. Quantum model for psychological measurements: from the projection postulate to interference of mental observables represented as positive operator valued measures. NeuroQuantology 12, 324-336 (2014).

[42] Yukalov, V.I.\& Sornette, D. Conditions for quantum interference in cognitive sciences. Top. Cogn. Sc. 6, 79-90 (2014). 
[43] Khrennikov, A. \& Basieva, I. Possibility to agree on disagree from quantum information and decision making. J. Math. Psych. 62(3), 1-5 (2014).

[44] Khrennikova, P. A quantum framework for 'Sour Grapes' in cognitive dissonance. In: Atmanspacher H., Haven E., Kitto K., Raine D. (eds) Quantum Interaction. QI 2013. Lecture Notes in Computer Science 8369 (Springer, BerlinHeidelberg, 2014).

[45] Busemeyer, J. R., Wang, Z., Khrennikov, A. \& Basieva, I. Applying quantum principles to psychology. Physica Scripta T163, 014007 (2014).

[46] Asano, M., Khrennikov, A., Ohya, M., Tanaka, Y.\& Yamato, I. Violation of contextual generalization of the Leggett-Garg inequality for recognition of ambiguous figures. Physica Scripta T163, 014006 (2014).

[47] Khrennikov, A., Basieva, I., Dzhafarov, E.N. \& Busemeyer, J. R. Quantum models for psychological measurements: An unsolved problem. PLOS ONE 9, Art. e110909 (2014).

[48] Boyer-Kassem, T., Duchene, S., Guerci, E. Quantum-like models cannot account for the conjunction fallacy. Theor. Decis. 10, 1-32 (2015).

[49] Dzhafarov, E. N., Zhang, R. \& Kujala, J. V. Is there contextuality in behavioral and social systems? Phil. Trans. Royal Soc. A 374, 20150099 (2015).

[50] Asano, M., Basieva, I., Khrennikov, A., Ohya, M., Tanaka, Y. \& Yamato, I. Quantum information biology: from information interpretation of quantum mechanics to applications in molecular biology and cognitive psychology. Found. Phys. 45, 1362-1378 (2015).

[51] Basieva, I. \& Khrennikov, A. On the possibility to combine the order effect with sequential reproducibility for quantum measurements. Found. Physics 45(10), 1379-1393 (2015).

[52] Khrennikov, A. Quantum-like model of unconscious-conscious dynamics; Frontiers Psych. 6, Art. N 997 (2015).

[53] Khrennikov, A. Quantum Bayesianism as the basis of general theory of decisionmaking. Phil. Trans. R. Soc. A 374, 20150245 (2016).

[54] Khrennikova, P. Quantum dynamical modeling of competition and cooperation between political parties: the coalition and non-coalition equilibrium model. $J$. Math. Psych. 71, 39-50 (2016). 
[55] Asano, M., Basieva, I., Khrennikov, A. \& Yamato, I. A model of differentiation in quantum bioinformatics. Prog. Biophys. Molecular Biol. Part A 130, 88-98 (2017).

[56] Yukalov, V.I.\& Sornette, D. Quantum probabilities as behavioral probabilities. Entropy 19, 112 (2017).

[57] Asano, M., Basieva, I., Khrennikov, A., Ohya, M. \& Tanaka, Y., A quantum-like model of selection behavior. J. Math. Psych. 78, 2-12 (2017).

[58] Khrennikova, P. Modeling behavior of decision makers with the aid of algebra of qubit creation-annihilation operators. J. Math. Psych. 78, 76-85 (2017).

[59] Basieva, I., Pothos, E., Trueblood, J., Khrennikov, A. \& Busemeyer, J. Quantum probability updating from zero prior (by-passing Cromwell's rule). J. Math. Psych. 77, 58-69 (2017).

[60] Khrennikov, A., Basieva, I., Pothos, E. M. \& Yamato, I. Quantum probability in decision making from quantum information representation of neuronal states, Sci. Rep. 8, 16225 (2018).

[61] Lawless, W. F. The interdependence of autonomous human-machine teams: The entropy of teams, but not individuals, Advances science. Entropy 21(12), 1195 (2019).

[62] Bagarello, F., Gargano, F. \& Oliveri, F. Spreading of competing information in a network. Entropy 22(10), 1169 (2020).

[63] Ozawa, M. \& Khrennikov, A. Application of theory of quantum instruments to psychology: Combination of question order effect with response replicability effect. Entropy 22(1), 37 (2020).

[64] Yukalov, V. I. Evolutionary processes in quantum decision theory. Entropy 22(6), 68 (2020).

[65] Penrose, R. The Emperor's new mind (Oxford Univ. Press, New-York, 1989).

[66] Umezawa, H. Advanced field theory: Micro, macro and thermal concepts (AIP, New York, 1993).

[67] Hameroff, S. Quantum coherence in microtubules. A neural basis for emergent consciousness? J. Cons. Stud. 1, 91-118 (1994).

[68] Vitiello, G. Dissipation and memory capacity in the quantum brain model. Int. J. Mod. Phys. B9, 973 (1995). 
[69] Vitiello, G. My double unveiled: The dissipative quantum model of brain, Advances in Consciousness Research. (John Benjamins Publ. Com., 2001).

[70] Ingarden, R. S., Kossakowski, A. \& Ohya, M. Information dynamics and open systems: Classical and quantum approach. Dordrecht, KLuwer, 1997).

[71] Benatti, F., Floreanini, R. (eds.) Irreversible quantum dynamics (Springer, BerlinHeidelberg, 2010).

[72] Ozawa, M. (1984). Quantum measuring processes for continuous observables; $J$. Math. Phys. 25, 79-87.

[73] von Helmholtz, H. (1866). Treatise on Physiological Optics. Transl. by Optical Society of America in English. New York, NY: Optical Society of America.

[74] Davies, E. B. Quantum Theory of Open Systems, Academic Press, London, 1976.

[75] Ozawa, M. (1997). An operational approach to quantum state reduction. Ann. Phys. (N.Y.) 259, 121-137.

[76] I. Basieva, A. Khrennikov, M. Ozawa, Quantum-like modeling in biology with open quantum systems and instruments. Biosystems 201, 104328 (2021).

[77] M. Ozawa, A. Khrennikov, Modeling combination of question order effect, response replicability effect, and QQ-equality with quantum instruments. J. Math. Psych. (2021).

[78] Giustina, M.; Versteegh, M.A.; Wengerowsky, S.; H.; steiner, J.; Hochrainer, A.; Phelan, K.; Steinlechner, F.; Kofler, J.; Larsson, J.Å; Abellan, C.; et al. A significant-loophole-free test of Bell's theo-rem with entangled photons. Phys. Rev. Lett. 2015, 115, 250401.

[79] James, W. (1890). The Principles of Psychology (New York: Henry Holt and Co.), Reprinted 1983 (Boston: Harvard Univ. Press).

[80] Jung, C. G. and Pauli, W. (2014). Atom and Archetype: The Pauli/Jung Letters 1932-1958, Princeton Univ. Press: Princeton.

[81] Freud, S. (1957). The Standard Edition of Complete Psychological Works of Sigmund Freud, Edited and Translated by J. Strachey, Vols. I-XXIV (The Hogarth Press, London).

[82] A. Yu. Khrennikov, Classical and Quantum Mental Models and Freud's Theory of Unconscious Mind. Växjö Univ. Press, Växjö, Sweden (2002). 
[83] Khrennikov, A. The quantum-like brain operating on subcognitive and cognitive time scales. J. Consciousness Studies 15, N 7, 39-77 (2008).

[84] Khrennikov A., Human subconscious as a p-adic dynamical system. J. Theor. Biol. 193(2), 179-96 (1998).

[85] Khrennikov A., Probabilistic pathway representation of cognitive information. J. Theor. Biol. 231(4), 597-613 (2004).

[86] A. Yu. Khrennikov, Toward an adequate mathematical model of mental space: Conscious/unconscious dynamics on $m$-adic trees. Biosystems 90 (3), 656-675 (2007).

[87] G. Iurato and A. Yu. Khrennikov, Hysteresis model of unconscious-conscious interconnection: exploring dynamics on $m$-adic trees. $p$-Adic Numbers Ultrametric Anal. Appl. 7 (4), 312-321 (2015). 